\title{
Schwangerschaft und Geburt
}

\section{Monika Sieverding}

Die Prävalenz der reproduktiven Risiken variiert weltweit sehr stark, wobei die Gesundheit von Frauen in Entwicklungsländern ungleich stärker bedroht ist. Dies wird am Beispiel der Müttersterblichkeit deutlich. Nach Angaben der WHO sterben jährlich 585.000 Frauen aufgrund von schwangerschaftsbedingten Komplikationen, wobei mehr als $98 \%$ dieser Todesfälle die Entwicklungsländer betreffen (Spicehandler, 1997). Die deutliche Verbesserung der reproduktiven Gesundheit von Frauen in den Industrienationen ist jedoch eine relativ junge Errungenschaft, zurückzuführen vor allem auf einschneidende medizinische Fortschritte, aber auch auf die Veränderung der Geschlechterrollen. Im Folgenden werden einige gesundheitsrelevante Aspekte, die mit Schwangerschaft und Geburt einhergehen, dargestellt.

\section{Abtreibung}

Die WHO schätzt, dass ungefähr jede 4. Schwangerschaft gezielt abgebrochen wird. Von geschätzten 45 Millionen weltweit durchgeführten Abtreibungen pro Jahr wird fast die Hälfte (ca. 20 Millionen) unter unsicheren und septischen Bedingungen durchgefuhrt, davon wiederum die Mehrheit in Entwicklungsländern. Illegale und unsichere Abtreibungen sind verantwortlich für ein weites Morbiditätsspektrum; im schlimmsten Fall enden sie tödlich (geschätzte Quote zwischen 50.000 und 100.000 Fälle pro Jahr). Dabei ist eine der wichtigsten Determinanten für das Mortalitätsrisiko die Legalität der Abtreibung. Aber auch die hohe Rate an legalen Schwangerschaftsabbrüchen beispielsweise in Ländern aus Zentral- und Osteuropa (dort war zumindest bis Anfang der 90er Jahre Abtreibung die häufigste Form der Geburtenkontrolle!) ist problematisch und hat ernsthafte Konsequenzen für die reproduktive Gesundheit von Frauen. Kurz- und längerfristige Folgen häufiger oder unter ungünstigen Bedingungen durchgeführter Abtreibungen in Osteuropa sind beispielsweise höhere Inzidenzen von Anämie, Infertilität und Frühgeburten. 


\section{Komplikationen im Zusammenhang mit Schwangerschaft und Geburt}

Komplikationen im Zusammenhang mit Schwangerschaft und Geburt - z. B. vorzeitige Wehen und Blutungen sowie Früh- und Fehlgeburten - sind für Frauen zwischen 15 und 45 Jahren der häufigste Anlass für eine stationäre Behandlung in einem Krankenhaus (Verbundprojekt, 2001). In Deutschland ist die Zahl der Schwangerschaften, die als „Risikoschwangerschaft" eingestuft werden, in den letzten Jahren ständig angestiegen; inzwischen werden zwei von drei Schwangerschaften als „Risikoschwangerschaft" eingestuft. Dass in anderen europäischen Ländern (z. B. Holland) diese Quote deutlich geringer ist (Schücking, 1994), verweist auf die Relativität des Risikobegriffs. Die von der Frauengesundheits-Forschung kritisierte Medikalisierung der Frauengesundheit scheint sich gerade auch auf diesem Gebiet niederzuschlagen (Schücking, 1994). Dies zeigt sich nicht zuletzt daran, dass - beispielsweise in den seit 1993 eingeführten Krankenhausdiagnosestatistiken - auch die normale Entbindung unter der ICD-Oberkategorie (630-679) „Komplikationen der Schwangerschaft, bei Entbindung und im Wochenbett" geführt wird.

\section{Fertilitätsstörungen}

Ein zunehmendes gesundheitsrelevantes Problem stellen Fertilitätsstörungen dar. Es wird geschätzt, dass weltweit ca. 50 bis 80 Millionen Menschen unter einer eingeschränkten Fertilität leiden. In Deutschland erlebt fast ein Drittel aller Frauen eine mindestens 12-monatige Episode der Unfruchtbarkeit (Huinink \& Brähler, 2000). Man nimmt an, dass 6 bis $9 \%$ aller Paare in Mitteleuropa ungewollt kinderlos sind und eine Behandlung wünschen.

In letzter Zeit wurde eine Palette moderner Techniken der assistierten Reproduktion entwickelt, die das Ziel haben, Fruchtbarkeitsstörungen eines Paares zu beheben: Beispiele sind die künstlichen Inseminationen mit dem Sperma des Partners oder eines Spenders oder In-Vitro-Fertilisationen (sogenannte Reagenzglas-Befruchtungen). Einhergehend mit der wachsenden Nachfrage nach Methoden der assistierten Reproduktion ist national wie international ein Trend mit einem rapiden Anwachsen reproduktionsmedizinischer Zentren zu beobachten.

Auch wenn diese Verfahren in vielen Fällen dazu beigetragen haben, den Kinderwunsch von infertilen Paaren $\mathrm{zu}$ erfüllen, sind sie nicht unproblematisch. So sind die zum Teil äußerst aufwendigen Behandlungsformen für viele Paare bei vergleichsweise bescheidenen Erfolgsquoten mit einer hohen 
psychischen Belastung verbunden. Bedenklich erscheint es auch, wenn versucht wird, psychisch (mit-)bedingte Unfruchtbarkeit mit medizintechnischen Mitteln zu „bekämpfen“.

\section{Das Konzept der reproduktiven Gesundheit}

Obwohl gesundheitliche Konsequenzen der menschlichen Reproduktion schon länger erforscht wurden, ist der Begriff ,,reproduktive Gesundheit“" relativ neu. Diese wurde 1994 auf der International Conference on Population and Development (ICPD) in Kairo zusammenfassend definiert: Danach beschreibt reproduktive Gesundheit einen Zustand vollständigen körperlichen, geistigen und sozialen Wohlbefindens in allen Belangen, die mit dem reproduktiven System bzw. seinen Funktionen und Prozessen zusammenhängen. Reproduktive Gesundheit bedeutet, dass Menschen ein befriedigendes und sicheres Sexualleben haben, sich fortpflanzen können und über die Entscheidungsfreiheit verfügen, ob, wann und wie oft sie sich reproduzieren. Während die sexuelle und reproduktive Gesundheit von Frauen in Entwicklungsländern vor allem durch ihre nichtgleichberechtigte Teilhabe am gesellschaftlichen Leben und ihre Abhängigkeit vom männlichen Partner eingeschränkt wird (Sieverding, 2001), zahlen viele Frauen aus westlichen Industriestaaten mit dem Verzicht auf Reproduktion einen hohen Preis für ihre berufliche und gesellschaftliche Emanzipation. Die gerade in Deutschland zunehmende Anzahl von Frauen, die entweder erst sehr spät oder gar keine Kinder bekommen, ist ein deutlicher Hinweis auf Einschränkungen der reproduktiven Gesundheit von Frauen. Und es wird ein weiterer Anstieg der Zahl kinderloser Frauen prognostiziert. So wird geschätzt, dass von der Geburtskohorte von 1965 jede dritte Frau kinderlos bleiben wird (Verbundprojekt, 2001). Beruflich engagierte Frauen wissen in diesem Land häufig nicht, wie sie die Anforderungen der Berufsrolle mit denen der Mutterrolle vereinbaren sollen. Das im internationalen Vergleich besonders schlechte Angebot an angemessenen (Ganztags-) Einrichtungen der Kinderbetreuung sowie eine nach wie vor nicht überwundene Mutter-Kind-Ideologie, die die Berufstätigkeit von Müttern (insbesondere kleinerer Kinder) negativ sanktioniert, macht Deutschland in dieser Hinsicht zu einem „Entwicklungsland“. 


\section{Literatur}

Huinink, J. \& Brähler, E. (2000). Die Häufigkeit gewollter und ungewollter Kinderlosigkeit. In E. Brähler, H. Felder \& B. Strauß (Hrsg.), Fruchtbarkeitsstörungen. Jahrbuch der Medizinischen Psychologie (Bd. 17, S. 43-54). Göttingen: Hogrefe.

Schücking, B. (1994). Schwangerschaft - (k)eine Krankheit? Zeitschrift für Frauenforschung, 12, 56-64.

Sieverding, M. (2001). Gender and reproductive health. In N. J. Smelser \& P. B. Baltes (Eds.), International Encyclopedia of Social and Behavioral Sciences (pp. 5969-5972). Oxford, England: Elsevier.

Spicehandler, J. (1997). Issues to consider in operationalizing reproductive health. Advances in Contraception, 13, 345-350.

Verbundprojekt zur gesundheitlichen Situation von Frauen in Deutschland (2001). Bericht zur gesundheitlichen Situation von Frauen in Deutschland (Schriftenreihe des Bundesministeriums für Familie, Senioren, Frauen und Jugend, Band 209). Stuttgart: Kohlhammer.

\section{Verweis auf andere Stichwörter}

$\rightarrow$ Frauengesundheit $\rightarrow$ Gesundheits- u. Krankheitsbegriffe $\rightarrow$ Klimakterium $\rightarrow$ Menstruation $\rightarrow$ Mortalität $\rightarrow$ Psychogynäkologie 